ISSN 1996-1073

www.mdpi.com/journal/energies

\title{
Article
}

\section{Expanding Horizons with Chameleon: Team Missouri's Innovative Home Automation System}

\section{Bryan Glass $^{1}$, Ben Brannon ${ }^{2}$, Katie Grantham ${ }^{3, *}$ and Stuart Baur ${ }^{4}$}

1 Department of Computer Engineering, Missouri University of Science and Technology, 1401 Pine Street, Rolla, MO 65409, USA; E-Mail: bmg262@mail.mst.edu

2 Department of Electrical Engineering, Missouri University of Science and Technology, 1401 Pine Street, Rolla, MO 65409, USA; E-Mail: btbfk2@mail.mst.edu

3 Department of Engineering Management, Missouri University of Science and Technology, 1401 Pine Street, Rolla, MO 65409, USA

4 Department of Civil, Architectural and Environmental Engineering, Missouri University of Science and Technology, 1401 Pine Street, Rolla, MO 65409, USA; E-Mail: baur@mst.edu

* Author to whom correspondence should be addressed; E-Mail: kag@ @mst.edu; Tel.: +1-314-580-0147.

Received: 7 April 2010; in revised form: 20 May 2010 / Accepted: 1 June 2010 /

Published: 4 June 2010

\begin{abstract}
Team Missouri's 2009 Solar Decathlon entry featured a revolutionary home automation system, Chameleon, promoting both convenience and energy savings. This was accomplished using the typical controls of a convenience based automation system, while maintaining user set points, such as a home's ambient temperature, in the most energy efficient manner. Environmental features of the system include controls for home heating, ventilation and air conditioning, lighting, windows, shades, appliances, indoor air quality, as well as indoor and outdoor irrigation. Further analysis and refinement to the prototype system displayed at the competition is also presented in this paper.
\end{abstract}

Keywords: residential; home automation; appliance control; energy conservation; optimization

\section{Introduction}

In recent years, significant research and development efforts have yielded progress in new methods of energy generation (e.g., improved wind turbines, higher efficiency solar cells, bio-based liquid fuels) 
and advancements to existing energy generation methods (e.g., cleaner burning coal fueled electricity plants, $\mathrm{CO}_{2}$ sequestration, nuclear power plant reliability). Fewer efforts have focused on consumer-level efficiency. After using traditional convenience-based automation systems in the 2007 Solar Decathlon, Team Missouri recognized the opportunity to realize energy savings through smart control algorithms implemented on the same hardware. Chameleon, Team Missouri's 2009 automation system, actively reduces energy consumption in buildings while still providing the convenience of an automatic control system.

Chameleon was developed as a growth platform for the continued investigation of energy management through typical convenience based automation systems. The system represents a paradigm shift in the manner of thinking about sustainability. Electronic devices have traditionally been viewed as energy consumers. Only recently has the feasibility of smart control for energy reduction been an intensive research area.

As a platform for continued research and development, primary emphasis was placed on system modularity. Controllers, modules, communication standards, input nodes, and feedback nodes were all developed to be individually upgradable. Further, code and algorithms were developed so that parameters will be updated as more efficient revisions are found. Quality control and error detection has been built into the code, as failure to communicate with a device or properly read a sensed value could significantly hinder results. Thus, while discussions of design, methodology, and performance are vital, further optimization is still ongoing and is not currently in the scope of the project detailed herein.

\subsection{Project Scope}

As the development time for the detailed system was constrained by third party deadlines, the scope of the project was limited to ensure successful completion of critical elements. Therefore, the components detailed in this paper include; component selection and interfacing, control methodology determination, and initial system test. Information regarding system efficiency, cost analyses, and lifecycle analyses are outside of the current scope and will be detailed upon collection of all necessary information.

\section{Results and Discussion}

\subsection{Background}

Currently, few commercial products exist that automate an entire building for both convenience and power conservation. Commercially available automation systems typically take each system as an individual unit and typically assume an unlimited power supply is available for each system. Energy management systems, on the other hand, typically control to limit energy usage without regard to individual creature comfort. Research implementations such as 'Home for Life' perform similarly to Chameleon, but are designed for a fixed research implementation and lack the modularity required for mass adoption and continued refinement [1]. Many building control systems are currently on the market. Siemens, Control Logic of Michigan, and Energy Control Systems Inc., for example, fall under the category of energy management systems (EMS). Home Automation Inc., Elan Home Systems, and HomeSeer fall under the category of home automation systems. Few bridge the gap in any manner. A 
project in Tokyo has set up a system that controls the air-conditioner using weather data provided from the internet, lights from occupancy and illumination sensors, and provides energy usage information feedback to the resident for particular appliances [2]. While the system provides comfort based on information and displays energy usage, it does not link the two together. ASHRAE's Technical Research Project 1286 [3] indicates a void of tools to assess efficiency and recommends that actions be taken to correct this. Chameleon furthers this by controlling a house utilizing this data to increase the efficiency.

Research efforts in academia and industry currently focus on furthering the technologies of the individual systems needed to enable a home automation system. For example, efforts have focused on the data congestion control problem that arises from the simultaneous communication between multiple residential systems with the home automation mainframe [4,5]. Also, reducing the energy required by the home automation system to run is currently being examined [6] as well as strategies to minimize the faults system faults [7].

During the 2009 competition several teams claimed to boast automation and energy management systems, so the following study, seen in Table 1, was completed to compare the various features and energy saving functions of all of the systems. As can be seen, most of the automation systems in the 2009 competition were simply data logging and energy feedback systems. There were a few true SCADA (supervisory control and data acquisition) systems that incorporated features such as lighting, HVAC, and window shade controls. The Chameleon system contains not only this standard set of abilities, but also controls windows, recovers waste heat from the bathroom and controls appliances.

\subsection{Team Missouri Home Automation System Design}

Two primary considerations were addressed throughout the development of Chameleon. First, the system would need to save energy while being seamless integrated into the home to encourage utilization. It would need to function as intended, but blend into the house as its name implied. Additionally, the system needed to be completely modular. As the system was a prototype under development its modular design allowed for greater flexibility. The controller chosen was built upon a reprogrammable Field Programmable Gate Array (FPGA) to allow unlimited reprogramming of any necessary algorithms. Additionally, the inputs and outputs were selected by placing expansion modules in a reconfigurable chassis. Inputs included sensor data (i.e., temperature, humidity, lighting level, solar irradiation, wind, air quality, occupancy, etc.) as well as user inputed data (i.e., house position and orientation, temperature and humidity preferences, privacy preferences, preferred schedule regarding appliance usage, etc.). Modules consisted of various high-level communication interfaces, as well as low level signal inputs, such as serial communications to the various system controllers to receive information and send commands. The reconfigurable chassis was able to accept any such module, and the configuration could be altered as needed. The two key components of Chameleon are the automation control system and the system model. 
Table 1. U.S. Department of Energy 2009 Solar Decathlon home automation features.

\begin{tabular}{|c|c|c|c|c|c|c|c|c|c|c|c|}
\hline Features & 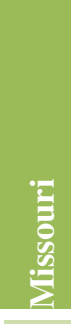 & 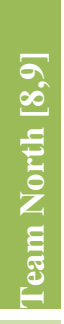 & 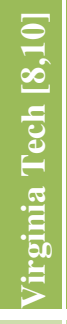 & 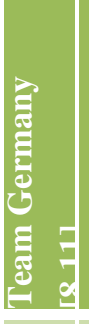 & 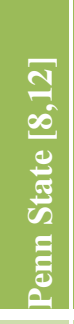 & 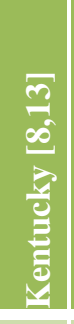 & 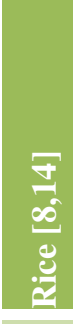 & 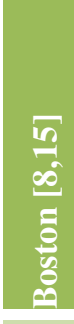 & 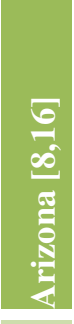 & $\begin{array}{l}\bar{E} \\
\frac{\infty}{0} \\
\overline{\overline{0}} \\
\overline{\bar{\theta}} \\
\dot{0}\end{array}$ & 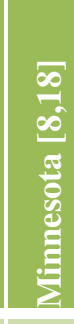 \\
\hline Data logging & $\mathrm{X}$ & $\mathrm{X}$ & $\mathrm{X}$ & $\mathrm{X}$ & $\mathrm{X}$ & & $\mathrm{X}$ & $\mathrm{X}$ & $\mathrm{X}$ & $\mathrm{X}$ & $\mathrm{X}$ \\
\hline Access Control & $\mathrm{X}$ & & $\mathrm{X}$ & & & & & & & $\mathrm{X}$ & \\
\hline User Customizable Entry & $\mathrm{X}$ & & & & & & & & & & \\
\hline Lighting Control & $\mathrm{X}$ & $\mathrm{X}$ & $\mathrm{X}$ & $\mathrm{X}$ & & & & & & & \\
\hline Appliance Control & $\mathrm{X}$ & & & & & & & & & & \\
\hline HVAC Control & $\mathrm{X}$ & $X$ & $\mathrm{X}$ & & & $X$ & & & & & \\
\hline Solar Domestic Hot Water Control & $\mathrm{X}$ & $\mathrm{X}$ & & & & $\mathrm{X}$ & & & & & \\
\hline Electric Usage Feedback & $\mathrm{X}$ & $\mathrm{X}$ & $\mathrm{X}$ & $\mathrm{X}$ & $\mathrm{X}$ & & & $\mathrm{X}$ & & $\mathrm{X}$ & \\
\hline Water Usage Feedback & $X$ & $\mathrm{X}$ & & & & & & $\mathrm{X}$ & & & \\
\hline Power Production Feedback & $\mathrm{X}$ & $\mathrm{X}$ & $\mathrm{X}$ & $\mathrm{X}$ & $\mathrm{X}$ & & & $\mathrm{X}$ & & $\mathrm{X}$ & \\
\hline Window Control & $\mathrm{X}$ & & & & & & & & & & \\
\hline Shade Control & $\mathrm{X}$ & $\mathrm{X}$ & & & & & & & & $\mathrm{X}$ & \\
\hline Irrigation & $\mathrm{X}$ & & & & & & & & & & \\
\hline Entertainment & $\mathrm{X}$ & & & & & & & & & $\mathrm{X}$ & \\
\hline Air Quality & $\mathrm{X}$ & & & & & & & & & & \\
\hline Energy Recovery Ventilator & $\mathrm{X}$ & & & & & & & & & & \\
\hline Mobile Device Interface & & & $\mathrm{X}$ & & & & & $\mathrm{X}$ & & & \\
\hline
\end{tabular}

\subsection{Chameleon Automation Control System}

To facilitate seamlessness to the end user, it was determined that Chameleon needed to be failsafe. To minimize rework and encourage future modularity, intermediate controllers were used, with the intelligent control being moved to the primary. Thus, logic, interfaces, and even the primary controller could be replaced as long as the same output events were mapped to the same output messages, much as in replacing a block in a blackbox model. This meant that were Chameleon to fail for any reason, all functions would remain active through the standard secondary controllers. The primary program is event driven, though events can be triggered internally by elapsed time. This implementation allows unneeded parts of the controller to sleep when idle, thus saving energy.

\subsection{System Flow}

The system uses set points and thresholds from the user, raw data from sensors, and processed data from other external sources when determining reaction states. It then processes the data on the primary controller to determine if corrective action is necessary, and outputs any user desired reaction to downstream controllers or directly to devices (Figure 1). 
Figure 1. Flowchart of Chameleon's operation.

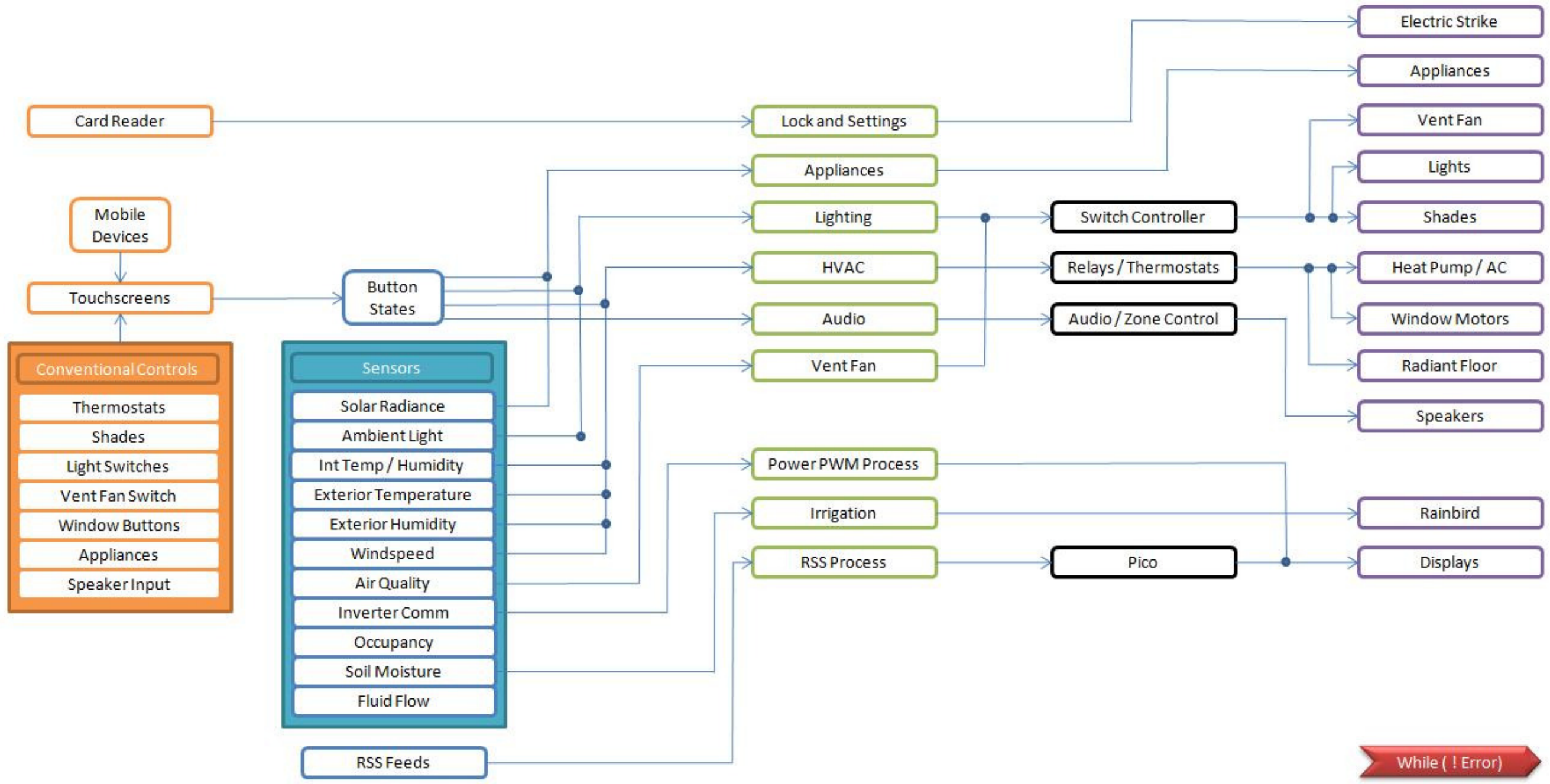


As demonstrated by the figure, the user retains all conventional controls throughout their home. They still have manually controllable thermostats, shades, light switches, vents, windows, appliances, and speakers. The automation system, however, also knows the state of these devices and will account for the current state when determining the actions to be taken. The user can additionally modify set-points and thresholds from a card reader, mobile devices, computers, and by interacting with touch-screens throughout the building.

All device states and settings are fed into the data network along with sensor output and parsed external communications. Due to the hierarchal nature of the program, it is then fed into an event driven processing script that contain the logic to optimize convenience, minimize energy, control devices, and modify device states and displays. Communication is bidirectional to this layer, so it may query output devices as needed or send setting information to input nodes as required.

\subsection{Chameleon Capabilities}

Chameleon receives input from computers, magnetic card readers, certain mobile devices, embedded local touch screens, and sensors. The system communicates with devices for three distinct purposes: convenience, energy optimization, and user education. Subsystems that can effect multiple areas attempt to balance their behavior by allowing users to set priorities, optimal values, and tolerances.

\subsubsection{Convenience}

As Chameleon envelopes the entire house, the first aspect that it controls is entry. A subroutine on the controller reads input from a magnetic card reader. If the user has clearance, the door is unlocked, and their settings are populated into the system. Currently, Chameleon tracks the user's favorite type of music, lighting preferences, temperature preferences, whether the user would like the lighting and temperature adjusted automatically upon entry, and greets the user by name. Additional users may be entered into the system during runtime, and temporary access can be granted based upon time of day or number of entries. While intended primarily for convenience, this functionality allows the user to turn off lights and HVAC systems upon leaving without having to worry about fumbling for controls upon return.

Similarly, a multi-input, multi-output speaker and zone control is included in the Audio/Video (A/V) system. A building may feature any number of inputs and output zones. This allows the resident to listen to an AV device anywhere in the house, or throughout the entire house, thus minimizing the amount of electronics required to be on (or even present).

Indoor plants are a staple of green building as they leech toxins from the air and improve indoor air quality. To encourage inhabitants to utilize indoor flora and allow them to be placed out of reach, an automatic irrigation system was developed. The system measures soil moisture content, and when it falls below threshold, opens a water valve. Additional air quality control is provided through automatic control of the vent fan. Air is circulated pursuant to ASHRAE recommended flow rates, and also based upon the calibrated threshold of an air quality sensor.

Finally, three low energy draw displays were installed throughout the house and the information was made available to other displays. This allowed the user to have RSS data such as weather, sports scores, e-mail, and news displayed through mirrors when getting ready for the day, or any other number 
of devices. This feature saved time, but also minimized the amount of time that high power draw devices such as computers and televisions had to be used.

\subsubsection{Energy Optimization}

While shades, windows, and lighting may be controlled for convenience, they also form the primary control devices for minimizing energy usage throughout the building.

One of the largest energy demands on a building is HVAC. Optimal temperature is maintained or achieved while minimizing energy usage through the monitoring of several conditions. If the combination of temperature and humidity outside is more favorable than that inside, windows will be opened to minimize the amount of time that typical air conditioning, heat pump, or radiant heat systems need to be used. Shades may also be opened. The thresholds for these actions were set based upon experimentally determined weighted scores, and the actions are executed if the instantaneous or short-period time averaged score exceeds these values. As temperature deviates from the set value, systems are engaged in a tiered structure based upon energy efficiency and user tolerances. The least energy intensive functions are engaged first, with step sizes indicative of the user's acceptable rate of correction. Further, heat and energy recovery ventilators can be controlled by the system to reclaim energy from exhaust airflows.

Though temperature may have an effect on shade state, light levels are the primary factor accounted for. Shades will be automatically opened and closed to maintain the optimal light level if the user has not overridden this system for privacy. Unnecessary lights in the house will be turned off or dimmed, but may also be turned on if the amount of ambient light is insufficient.

High power-draw appliances account for approximately $27 \%$ of residential power usage as shown in Chart 2 (12). Significant work has been done to increase the internal efficiency of appliances by manufacturers, but balancing building usage and running appliances at the optimal time is an opportunity for further energy savings. Through collaboration with Whirlpool, a washing machine, dryer, and dishwasher that could receive control commands from an external source were implemented. The user prepared the appliances to run, queued them through a touchscreen interface, and Chameleon would then determine the optimal time to start the cycle. As many homes are equipped with solarthermal collectors, the option was available to run appliances at the time of highest solar irradiance when hot water could be produced without requiring energy. If not present, the system ran at off peak hours to minimize peak usage charges and balance the energy demand of the house.

\subsubsection{User Education}

Minimizing the envelope of a building is a crucial step, but educating the user is equally important for long term energy minimization. As such, Chameleon made the user aware of their habits and the corresponding impact. Energy production and energy usage were both monitored and displayed. Additionally, a net balance was calculated, and displayed in terms of the financial impact to the user based upon current energy price.

\subsection{Control}

Making Chameleon easy to control is crucial to it being adopted. The touch-screens throughout the home were selected and programmed to run a simple yet powerful program that was pleasing to the 
user. Tabs were used to organize similar tasks and keep the interface from appearing cluttered (Figure 2). A consistent background was used across all tabs so that information could be located easily. All traditional home controls remained and were as failsafe as if Chameleon were not present. In the event that the primary controller was taken offline, traditional controls would either communicate with intermediate controllers or with the devices that they were controlling directly.

Figure 2. Average residential energy use [19].

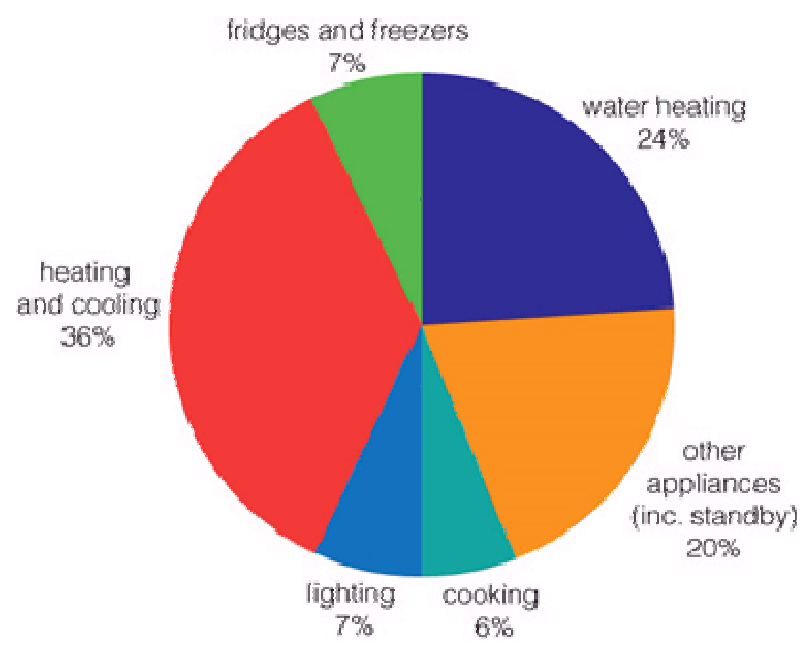

The mobile device interface was designed using the same theme as the Human Machine Interface (HMI) running on the touch-screens, see Figure 3. The newly developed communication interface for mobile devices, however, provides an application programming interface (API) that will allow any interface (or none at all for devices that are not capable of running applications) to be used.

Figure 3. Appliance tab from in-home Human Machine Interface.

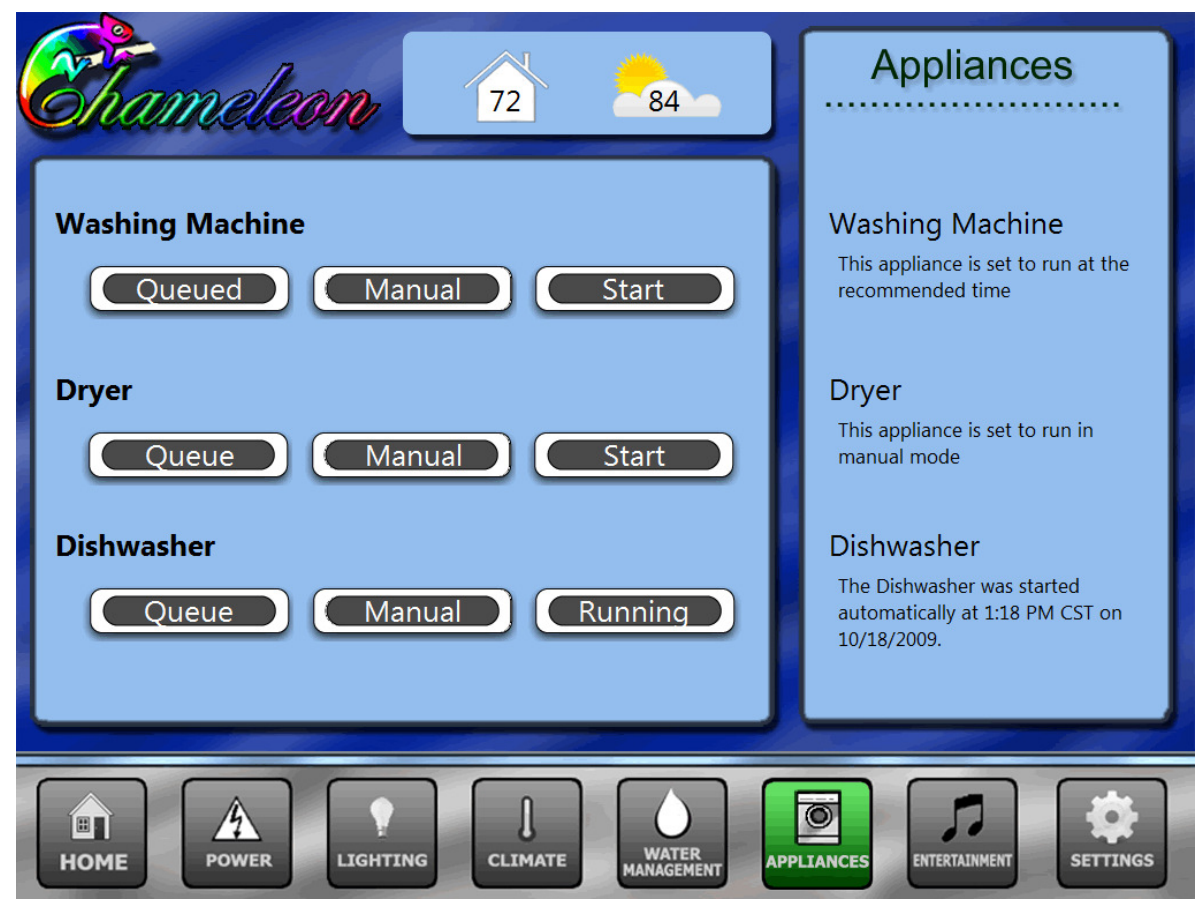




\subsection{Impact on Consumer}

The positive impact of this project on the electric consumer is high. This system provides a reduction in energy consumption, achieves cost savings by running high-energy appliances during off-peak hours, educates the consumer as to what their real-time energy consumption is through a graphical interface, all while maintaining the comfort level that the user specified in his or her personal preferences. The main goal of this project was to decrease energy consumption using a system that is transparent to the end-user.

\section{Experimental Section}

Chameleon was unveiled to the public at the 2009 Solar Decathlon in Washington, D.C. Over 21,000 members of the public, as well as several government officials and industry experts, toured the house and interacted with the system. It ran without error for the entire competition period, and received all positive reviews from judges. Competition rules required systems to run at certain times, so much of the automated control was disabled throughout parts of competition. Thus, the data obtained on energy usage did not yield conclusive results on the impact of the system. The simulated values and energy models discussed will be verified upon reinstallation of the home on its permanent site.

\section{Conclusions}

The initial run of the Chameleon system covered a seven-day period, executed 3,271 actions, and read 14,843 sensor values. While it is likely that not all values and serial strings were transmitted without error, no communication errors resulted, thus indicating that the internal event handling of the system functioned as intended. With no external communication errors in 18,114 opportunities, the DPMO and subsequent sigma rating indicate that the system performed above typical industry expectations. The small sample size and short test period hinder statistical analysis, but encourage further test.

While the cost of the prototype system used is likely far more expensive than a future retail implementation. The total prototype cost of $\$ 33,818.70$ includes, control systems, intermediate controllers, sensors, actuators, end effectors, input nodes, and informational displays. Much of this cost is dedicated to extra equipment for testing and system development which is unnecessary in a simple residential application by the consumer.

As Missouri S\&T has four solar homes with different designs and varying degrees of automated control and data acquisition, the research group is well poised to perform various types of research and development.

\subsection{Evaluation Laboratory-S\&T Solar Village}

The scope of this project includes a feasibility study of Chameleon focused on measuring the energy usage between similar homes-with and without automation systems. The S\&T Solar Village is the resting place for its Solar Decathlon competition entries. The village layout is shown in Figure 4. Each house serves as student housing providing an opportunity to measure real world conditions and compare different energy systems with similar homes. The solar and thermal systems controllers are 
placed in the basement of each house and therefore are accessible without disturbing the resident and the living environment.

Figure 4. Missouri S\&T solar village.

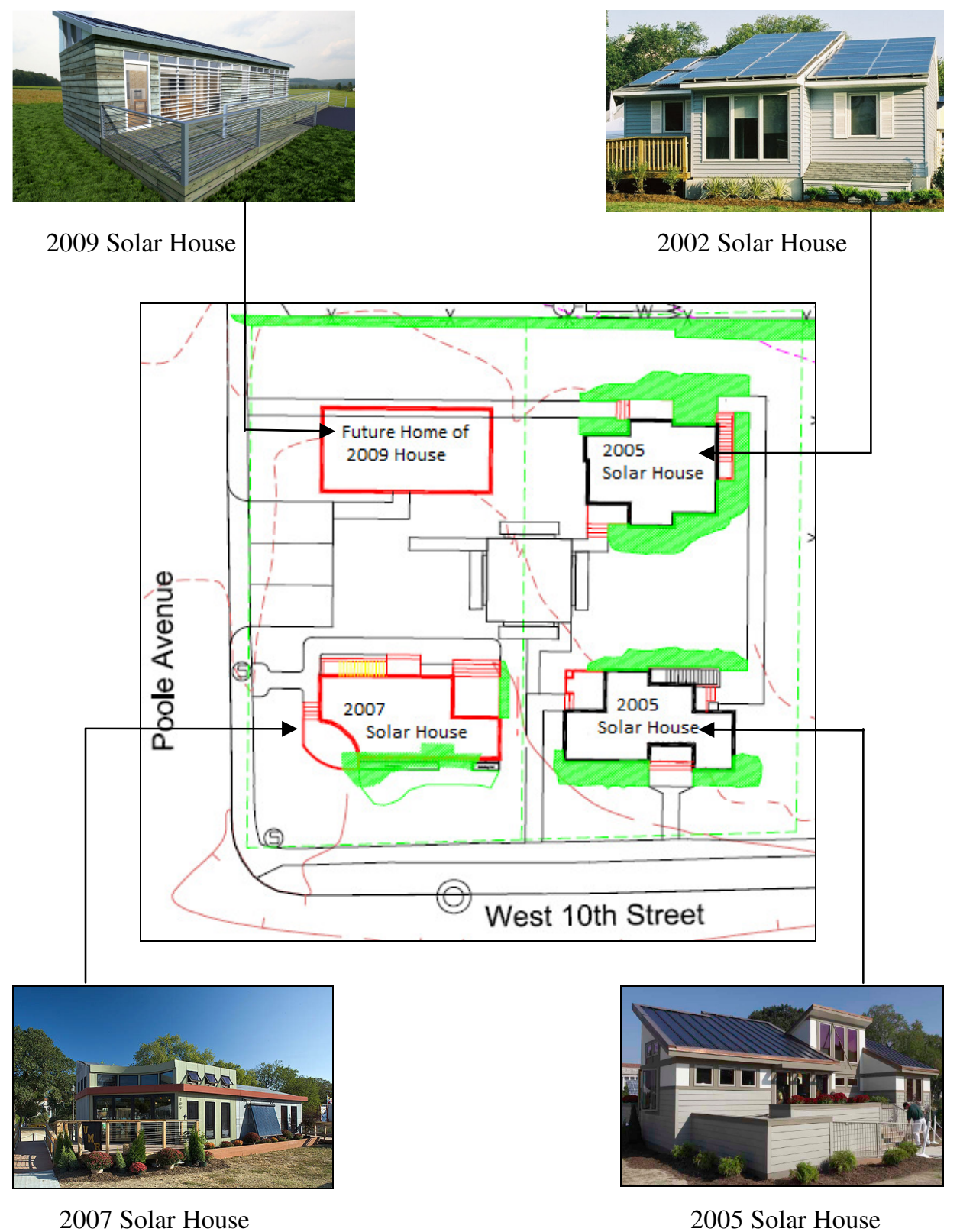

Upon the re-installation of the system, a set of experiments will be run to quantify the energy savings of the Chameleon system. The experiments will run for one year, which provides a unique opportunity to capture real-world usage data. To date, 130 sensors have been installed in the three standing houses of the solar village (see Figure 4). Through our partnership with NREL, approximately $30 \%$ of the sensors have been installed and are collecting data. S\&T is continuing to work with NREL to complete the sensor installation by August 2010. The $30 \%$ of installed sensors are those that measure the Alternating Current (AC). These were installed in December of 2008 in all three homes and the data collection system has been operating since January $18^{\text {th }}$ of 2009 . The data is being stored in a database for long-term collection and analysis. 
A feedback interface was created to monitor the homes. The Internet accessible feedback displays the pseudo real-time ( 1 minute delay) energy demand and the access day's total energy usage for all three instrumented solar homes. Currently, work is in progress to make the data interface more intuitive to general users as well as assist with automated analysis needed to support the Chameleon project.

\subsection{EPA SBIR}

The group responsible for developing Chameleon will be working with Interdisciplinary Design Collaborative (IDC), a student company on the Missouri S\&T campus, in fulfillment of an SBIR awarded by the EPA. Data will be collected, logged, and analyzed to determine algorithms for the following:

i. Ten system controls that that have a high potential for improvement in residential energy conservation

ii. Five system controls that have a high potential for improvement in indoor air quality

iii. Heating and air conditioning performance metric determination

iv. Maximum HVAC efficiency based on power consumption and performance

v. Energy production predictions based on a photovoltaic system and weather forecasts

vi. The determination of optimum times to run a dishwasher, clothes washer, and clothes dryer

vii. Defined conditions in which it is more efficient to utilize passive cooling (versus active cooling)

By setting sensors in sample homes and collecting data correlating to energy consumption over time, generalized algorithms will be programmed to optimize energy consumption in buildings. These algorithms will be tested to assure greatest optimization.

\subsection{System Reduction}

Concurrent to the optimization and logging detailed in sections 4.1 and 4.2, the prior Chameleon automation system will be refined. This project will focus on five key areas: (1) the interfacing of additional communication standards to facilitate the use of a wider array of devices, (2) control rectification, (3) detailed energy study of all components to be used, (4) a reduction in system power consumption (based upon the data obtained and the minimization of transformers and leakage), and (5) a final study to analyze all results. Upon completion and verification of the results, automated black box testing will commence to ensure that the system exceeds the industry defect rate.

\section{References and Notes}

1. Active House-Home for Life. Available online: http://www.velfac.dk/Global/Home_for_life (Accessed on 20 May 2010).

2. Kushiro, N.; Suzuki, S.; Nakata, M.; Takahara, H.; Inoue, M. Integrated residential gateway controller for home energy management system. IEEE Trans. Consum. Electron. 2003, 49, 629-636.

3. Glazer, J. Evaluation of Building Energy Performance Rating Protocols; ASHRAE 1286-TRP; ASHRAE Technical Committee TC 7.6-Systems Energy Utilization: Atlanta, GA, USA, 2006. 
4. Lee, D.; Chung, K. Adaptive duty-cycle based congestion control for home automation networks. IEEE Trans. Consum. Electron. 2010, 56, 42-47.

5. Pungila, C.; Fortis, T.; Arvitan, O. Benchmarking database systems for the requirements of sensor readings. IETE Tech. Rev. 2009, 26, 342-349.

6. Park, W.; Choi, C.; Lee, I.; Jang, J. Energy efficient multi-function home gateway in always-on home environment. IEEE Trans. Consum. Electron. 2010, 56, 106-111.

7. Moon, K.; Ha, Y. An error messages clustering-based fault management framework for adaptive home network middlewear. IEEE Trans. Consum. Electron. 2010, 56, 63-69.

8. Smith, P. Survey of Home Monitoring Control Systems at the Solar Decathlon 2009. Available online: http://patricksmith.org/blog/2009/10/survey-of-home-monitoring-controlsystemsat-thesolar-decathlon-2009 (Accessed on 14 November 2009).

9. Engineering Design. Available online: www.team-north.com (Accessed on 20 May 2010).

10. House Management System User Interface. Available online: www.solar.arch.vt.edu (Accessed on 20 May 2010).

11. Active Technologies. Available online: www.solardecathlon2009.de (Accessed on 20 May 2010).

12. Live Monitoring. Available online: www.naturalfusion.org (Accessed on 20 May 2010).

13. Something Old, Something New, Something Blue. Available online: www.uky.edu/solarhouse (Accessed on 20 May 2010).

14. Zerow House. Available online: www.ricesolardecathlon.org (Accessed on 20 May 2010).

15. Feedback. Available online: www.livecurio.us (Accessed on 20 May 2010).

16. Seed [pod]. Available online: www.uasolardecathlon.com (Accessed on 20 May 2010).

17. Home Automation, Security, and entertainment. Available online: www.cusd.cornell.edu (Accessed on 20 May 2010).

18. Design Factors. Available online: www.solardecathlon.umn.edu (Accessed on 20 May 2010).

19. Be Energy Smart. Department for Transport, Energy and Infrastructure, Government of South Australia, 2009. Available online: http://www.dtei.sa.gov.au/energy/be_energy_smart (Accessed on 20 May 2010).

(C) 2010 by the authors; licensee MDPI, Basel, Switzerland. This article is an Open Access article distributed under the terms and conditions of the Creative Commons Attribution license (http://creativecommons.org/licenses/by/3.0/). 